\title{
Vigilancia de los niveles de uso de antibióticos y perfiles de resistencia bacteriana en hospitales de tercer nivel de la Ciudad de México
}

Lilia Benavides-Plascencia, $M$ en $C,{ }^{(1)}$ Alejandro Leonardo Aldama-0 jeda, $M$ en $C,{ }^{(2)}$ Héctor Javier Vázquez, Dr en C.(2)

\section{Benavides-Plascencia L,Aldama-Ojeda AL,Vázquez HJ. Vigilancia de los niveles de uso de antibióticos y perfiles de resistencia bacteriana en hospitales de tercer nivel de la Ciudad de México. Salud Publica Mex 2005;47:219-226.}

\section{Resumen}

Objetivo. Determinar los niveles de uso de antibióticos y el perfil de resistencia de las bacterias nosocomiales, e identificar y proponer estrategias para disminuir la resistencia a los antibióticos. Material y métodos. Estudio descriptivo, retrolectivo (1994-1995), hecho en seis hospitales de tercer nivel de la Ciudad de México. Resultados La resistencia del grupo hospitalario fue de $86 \%$, mientras que el consumo total de antibióticos por institución varió entre 44 y 195 dosis diarias definidas/100 camas-día. Conclusiones. Se identificaron los elementos para instrumentar un sistema de vigilancia integral que mejore el uso de antibióticos y la valoración de la resistencia bacteriana.

Palabras clave: antibióticos; resistencia microbiana a las drogas; México

\author{
Benavides-Plascencia L,Aldama-Ojeda AL,Vázquez HJ. \\ Surveillance of antibiotic utilization \\ and bacterial resistance profiles \\ in tertiary level hospitals in Mexico City. \\ Salud Publica Mex 2005;47:219-226.
}

\section{A bstract}

Objective.To identify the levels of antibiotic utilization and the resistance profiles of nosocomial bacteria, as well as the strategies to diminish resistance to antibiotics. Material and methods A descriptive, retrospective (1994-1995) study was conducted in six tertiary level hospitals in Mexico City. Results. A total of $86 \%$ antibiotic resistance was observed in these hospitals. The overall consumption of antibiotics per hospital ranged between 44 and 195 Defined D aily D oses/100 day-beds. Conclusions. W e identified the components to frame an integral surveillance system aimed at improving the use of antibiotics and the quality of the bacterial resistance assessment in these hospitals.

Key words: antibiotics; resistance microbial; Mexico

(1) Departamento de Sistemas Biológicos, DCBS, UAM-X Universidad Autónoma Metropolitana, Unidad Xochimilco. México, DF, México.

(2) Departamento de Sistemas, D C BI, UAM-A, Universidad Autónoma Metropolitana, U nidad Azcapotzalco. México, DF, México.

Fecha de recibido: 1 de julio de 2004 • Fecha de aprobado: 11 de abril de 2005

Solicitud de sobretiros: Lilia Benavides Plascencia. Universidad Autónoma Metropolitana-X ochimilco, D irección de Ciencias Biológicas y de la Salud. Calzada del Hueso 1100, coloniaVilla Q uietud, 04960 México, DF, México.

Correo electrónico: benavidl@ correo.xoc.uam.mx 
E 1 problema de la resistencia a los antibióticos es global, complejo, incluye un gran número de especies bacterianas de importancia médica y es de difícil control por su multicausalidad. El consumo masivo de antibióticos en los últimos 50 años ha creado un ambiente favorable a la selección de bacterias que soportan los efectos tóxicos de los antimicrobianos. ${ }^{1}$ Los cambios en la ecología de las infecciones nosocomiales observadas en los hospitales desde la introducción de los agentes antimicrobianos han sido ampliamente documentados. ${ }^{2}$ Entre los factores que han contribuido al aumento de la resistencia a los antibióticos están la concentración de la población en centros urbanos, el inadecuado control de las infecciones en los hospitales, la tendencia a internar en hospitales a los pacientes seriamente enfermos, la migración masiva a través de las regiones del globo y el uso inadecuado de los antibióticos, entre otros. ${ }^{3}$

Las bacterias patógenas de la época preantibióticos eran raramente resistentes. ${ }^{4}$ Actualmente $70 \%$ de las bacterias responsables de las infecciones nosocomiales son resistentes al menos a uno de los antibióticos más comúnmente utilizados para tratarlas. ${ }^{5} \mathrm{El}$ uso irracional de los antimicrobianos ha contribuido al aumento en la resistencia bacteriana. ${ }^{6}$ Las bacterias se adaptan rápidamente a las condiciones de su medio, aun en la presencia de estos fármacos. Los antibióticos difieren de los otros medicamentos porque no sólo ejercen un efecto terapéutico sino que alteran también la ecología de la microflora del cuerpo y del medio externo. ${ }^{7}$ La gran capacidad adaptativa de las bacterias es el resultado del efecto combinado de rápidos índices de crecimiento, de mutaciones genéticas y de la selección de las mismas, así como de su habilidad para intercambiar material genético horizontalmente. ${ }^{8,9}$

En las últimas dos décadas se han incrementado las investigaciones para explorar las causas y las formas de controlar o prevenir la resistencia a los antibióticos. Los estudios del uso de antibióticos y la resistencia bacteriana a los antimicrobianos asociada al uso se han encontrado con problemas que se relacionan con los métodos para investigarlos. ${ }^{10,11}$ Estudios con base en diseños epidemiológicos tradicionales demostraron distintos grados de asociación entre la resistencia a un antibiótico particular y sus niveles de consumo. ${ }^{12-17} \mathrm{Sin}$ embargo, el fenómeno de la resistencia a los antibióticos es complejo y requiere de hipótesis, análisis y metodologías innovadoras. ${ }^{18-23}$

La vigilancia continua del uso de antibióticos y del perfil de resistencia bacteriana a estos medicamentos es un área relativamente nueva. Debido a la importancia del problema se han integrado grupos de trabajo multidisciplinarios que han emitido recomendaciones a escala local ${ }^{24-27}$ e internacional ${ }^{28-30}$ para desarrollar planes tendientes a disminuir la resistencia a los antimicrobianos.

La Organización Mundial de la Salud (OMS) publicó, en septiembre de 2001, su WHO Global Strategy for Containment of Antimicrobial Resistance, ${ }^{30}$ como resolución de la Asamblea Mundial de la Salud en 1998, e invitó a los países miembros a la adopción de medidas para limitar la diseminación de la resistencia a los antibióticos. Se propuso entonces la inclusión de la vigilancia de la resistencia a los antibióticos, y la obligatoriedad del reporte sobre resistencia a estos fármacos en las revisiones de las regulaciones internacionales de la salud.

Los objetivos del presente trabajo son determinar los niveles del uso de antibióticos y el perfil de la resistencia bacteriana a los antibióticos en seis hospitales de los institutos nacionales de salud en la Ciudad de México (HINSCM), así como describir el estado de la vigilancia de la resistencia bacteriana y del uso de antibióticos en esos hospitales, e identificar y proponer estrategias para disminuir la resistencia.

\section{Material y métodos}

Se trata de un estudio transversal, retrolectivo y multicéntrico, cuya población objetivo fueron seis hospitales de tercer nivel pertenecientes al sector salud, que aceptaron participar. Se seleccionó al sector público por la facilidad de acceder a la información y por su gran cobertura en la Ciudad de México.

Se tomó una muestra de conveniencia por cuotas, ${ }^{31}$ de 100 casos de infección nosocomial por institución, correspondientes al periodo 1994-1995, y la información sobre identidad y sensibilidad a los antibióticos de los aislamientos bacterianos detectados en los mismos (el primo aislamiento de cada cepa por paciente). Los datos se captaron mediante cinco formatos específicos por instancia hospitalaria, en las modalidades de cuestionarios, registros de laboratorio y entrevista: forma I para la administración del hospital, formas II y III para el laboratorio de microbiología diagnóstica, forma IV para el comité de infecciones y forma $\mathrm{V}$ para la farmacia. Se dejaron los cuestionarios autoadministrables y se completó la información con una entrevista a los responsables de cada instancia; salvo la forma III, que la completó el investigador con la información recabada en el laboratorio a partir de la revisión de los registros de resultados en cuadernos o en la computadora. Las instituciones participantes se identificaron con una clave por acuerdo previo de confidencialidad.

En el presente trabajo se consideró: a) uso de antibióticos: dosis diarias definidas por 100 camas-día $^{32}$ 
(DDD/cd), de cada antibiótico dispensado en la farmacia del hospital respectivo, y número de antibióticos distintos disponibles en la farmacia; para el cálculo de las DDD/100 cd por antibiótico, se emplearon las cantidades de cada antibiótico adquiridas por la institución en el periodo estudiado; se hizo la conversión de las unidades a gramos de principio activo, según la presentación y con base en la información por medicamento del Diccionario de Especialidades Farmacéuticas 2002,33 b) resistencia: porcentaje de cepas resistentes por hospital en relación con los aislamientos considerados; se estimó el número de géneros resistentes por hospital, y el número de cepas resistentes por antibiótico valorado, y c) multirresistencia: número de grupos de antibióticos a los que es resistente una cepa bacteriana.

El análisis estadístico de los datos se llevó a cabo mediante gráficas de pareto, y análisis de conglomerados jerárquico y de componentes principales.

\section{Resultados}

\section{Uso de antibióticos}

El número de antibióticos dispensados en la farmacia y el porcentaje de éstos incluidos en las pruebas de sensibilidad en el laboratorio para cada hospital fueron: $\mathrm{A}(26 / 88 \%), \mathrm{B}(15 / 73 \%), \mathrm{C}(22 / 77 \%), \mathrm{D}(27 / 65 \%)$, E (22/66\%) y F (18/76\%). Los grupos de antibióticos con mayor consumo, en DDD/100 cd, en los seis hospitales fueron: cefalosporinas (216.1), betalactámicos (127.7), aminoglucósidos (86.9), sulfonamidas (36.1), lincosamidas (27.6), imidazoles (22) y quinolonas (19.6). Los menos consumidos fueron: tetraciclinas $(0.2)$, monobactámicos (0.9), nitrofuranos (1.7) y anfenicoles (1.8).

Los hospitales con un consumo mayor en DDD/ 100 cd fueron: D (194.8), B (133.4) y A (81.2); mientras que $\mathrm{F}$ (100), C (97.8) y A (86.5) presentaron el mayor porcentaje de cepas $\mathrm{R}$.

De acuerdo al consumo y como resultado de los análisis de conglomerados jerárquico y de componentes principales, se conformaron tres grupos de antibióticos: los de bajo nivel [con DDD/100 $\mathrm{cd} \leq 1$ ], los de nivel medio [con DDD/100 $\mathrm{cd} \leq 10$ ] y los de alto nivel [con DDD/ $100 \mathrm{~cd}>10$ ] (cuadro I).

Al analizar la resistencia por antibiótico, fueron siete los responsables de $80 \%$ de la resistencia observada en los seis hospitales estudiados: gentamicina $(\mathrm{Gm})$, ampicilina (Am), ciprofloxacina (Cip), amikacina (Ak), cefalotina (Cf), piperacilina (Pip) y trimetoprim-sulfametoxazol (T/S). Dos aminoglucósidos, Ak y Gm, presentaron la resistencia más alta $(>20 \%)$ en cuatro de las seis instituciones. La resistencia a la Cip
Cuadro I

\section{Niveles de Consumo de ANTIBióticos CON NÚmero DE CEPAS RESISTENTES EN LOS HOSPITALES ESTUDIADOS. Ciudad de MÉxico, DF, 1995}

\begin{tabular}{|c|c|c|c|}
\hline $\begin{array}{l}\text { Nivel de consumo } \\
\text { de antibióticos }\end{array}$ & $\begin{array}{c}\text { Tipo de } \\
\text { antibiótico }\end{array}$ & $\begin{array}{l}\text { DDD/ } \\
100 \mathrm{~cd}\end{array}$ & $\begin{array}{l}\text { Número de } \\
\text { cepas resistentes }\end{array}$ \\
\hline \multirow[t]{5}{*}{ Bajo } & Tetraciclinas & 0.20 & 1 \\
\hline & Monobactámicos & 0.30 & 7 \\
\hline & Anfenicoles & 0.45 & 4 \\
\hline & Carbapenems & 0.60 & 12 \\
\hline & Glucopéptidos & 0.97 & 1 \\
\hline \multirow[t]{5}{*}{ Medio } & Macrólidos & 1.60 & 10 \\
\hline & N itrofuranos & 1.70 & 1 \\
\hline & Quinolonas & 3.90 & 51 \\
\hline & Lincosaminas & 5.50 & 8 \\
\hline & Sulfonamidas & 6.00 & 20 \\
\hline \multirow[t]{3}{*}{ Alto } & Aminoglucósidos & 14.40 & 56 \\
\hline & Betalactámicos & 21.20 & 131 \\
\hline & Cefalosporinas & 36.00 & 117 \\
\hline
\end{tabular}

DDD/100 cd: dosis diarias definidas/100 camas-día

sobrepasó 30\% en tres de los hospitales estudiados (cuadro II).

Perfil de resistencia de las bacterias nosocomiales

Del total de 669 aislamientos bacterianos, $86 \%$ fue resistente a alguna clase de antibiótico (cuadro III).

La identificación de las cepas en los hospitales se realizaba en general hasta la especie. El número de cepas que se señalaron como no identificadas fue muy bajo. Se detectaron 19 géneros bacterianos resistentes en este grupo de hospitales: Acinetobacter, Branhamella, Citrobacter, Haemophilus, Moraxella, Proteus, Salmonella, Serratia, Staphylococcus, Streptococcus y Xanthomonas. Enterobacter, Enterococcus, Escherichia, Klebsiella y Pseudomonas estuvieron presentes en las seis instituciones. Mientras que Alcalígenes, Flavobacterium y Shigella se encontraron en un solo hospital. Acinetobacter, Enterobacter, Enterococcus, Escherichia, Klebsiella, Pseudomonas, Staphylococcus, Streptococcus y Xanthomonas, resultaron responsables de $80 \%$ de la resistencia observada, y variaron en frecuencia según el hospital. En el hospital F, dos géneros, Pseudomonas y Staphylococcus, constituyeron $84.7 \%$ de la resistencia observada en ese nosocomio. 


\section{Cuadro II \\ RESISTENCIA MICROBIANA A LAS DROGAS Y NIVEL DE CONSUMO DE ANTIBIÓtICOS, POR HOSPITAL. CiUdad de MéXICO, DF, 1995}

\begin{tabular}{|c|c|c|c|c|c|c|c|c|c|c|c|c|}
\hline \multirow[b]{3}{*}{ Tipo de antibióticos } & \multicolumn{12}{|c|}{ Hospitales } \\
\hline & \multicolumn{2}{|c|}{ A } & \multicolumn{2}{|c|}{$B$} & \multicolumn{2}{|c|}{ C } & \multicolumn{2}{|c|}{$\mathrm{D}$} & \multicolumn{2}{|c|}{$\mathrm{E}$} & \multicolumn{2}{|c|}{$\mathrm{F}$} \\
\hline & $\overline{D D D}$ & $\overline{C R}$ & $\overline{\mathrm{DDD}}$ & $\overline{C R}$ & $\overline{\mathrm{DDD}}$ & $\overline{C R}$ & $\overline{\mathrm{DDD}}$ & $\overline{C R}$ & $\overline{\mathrm{DDD}}$ & $\overline{C R}$ & $\overline{D D D}$ & $C R$ \\
\hline Tetraciclinas & 0.2 & 1 & & & & 14 & & 2 & & 8 & & \\
\hline Amfenicoles & 0.5 & 4 & & & 0 & 1 & 1.3 & 1 & 0 & & 0 & 11 \\
\hline$\beta$ lactámicos & 10.9 & 168 & 13.2 & 115 & 10.0 & 134 & 75.6 & 166 & 5.8 & 82 & 12.2 & 125 \\
\hline Cefalosporinas & 22.9 & 108 & 111.1 & 84 & 24.2 & 222 & 23.3 & 92 & 21.2 & 64 & 13.4 & 135 \\
\hline Monobactámicos & 0.6 & 18 & & & 0.2 & 3 & 0.1 & 1 & & 2 & & 2 \\
\hline Carbapenems & 1 & 7 & 0.3 & & 0.5 & 29 & & 33 & 0.3 & 2 & & 12 \\
\hline Sulfonamidas & 3.1 & 22 & 2.3 & 22 & 1.3 & 20 & 27.4 & 37 & 1.6 & 16 & 0.7 & 6 \\
\hline Macrólidos & 0.2 & 12 & & 8 & 1.2 & 25 & 5.4 & 3 & 0.7 & 7 & 0.5 & 3 \\
\hline Lincosamidas & 3.5 & 8 & & 2 & 0.6 & 16 & 15.5 & 2 & 4.0 & 8 & 4.0 & 7 \\
\hline Aminoglucósidos & 10.3 & 101 & 4.6 & 30 & 6.6 & 58 & 38.3 & 131 & 14.6 & 8 & 12.5 & 8 \\
\hline Q uinolonas & 7.6 & 67 & 1,0 & 32 & 2.7 & 26 & 3.8 & 125 & 4.5 & 6 & & 6 \\
\hline Glucopéptidos & 2.3 & 1 & 0.8 & & 1.1 & & 0.3 & 2 & 0.7 & 1 & 0.6 & 1 \\
\hline Imidazoles & 15.4 & & & & 0.3 & & 3.8 & & 2.4 & & 0.1 & \\
\hline
\end{tabular}

DDD: dosis diarias definidas por 100 camas-día $\mathrm{CR}$ : cepas resistentes

N ota: Las celdas vacías indican la inexistencia del dato

Cuadro III

RESISTENCIA MICROBIANA A LAS DROGAS POR HOSPITAL, Y POR EL TOTAL DE LA MUESTRA. Ciudad de México, DF, 1995

Aislamientos Aislamientos Total \% resistencia \% resistencia Hospital resistentes sensibles cepas en el hospital de la muestra

\begin{tabular}{rrrrrr} 
A & 135 & 21 & 156 & 86.5 & 23.43 \\
\hline B & 109 & 31 & 140 & 77.8 & 18.92 \\
\hline C & 92 & 2 & 94 & 97.8 & 15.97 \\
\hline D & 126 & 31 & 157 & 80.2 & 21.87 \\
\hline E & 42 & 8 & 50 & 84.0 & 7.29 \\
\hline F & 72 & 0 & 72 & 100.0 & 12.50
\end{tabular}

Total

576

93

669

99.98

Los antibióticos contra los que presentaron resistencia las principales cepas Gram positivas y negativas en el grupo de hospitales se muestra en el cuadro IV.

La mayoría de las cepas mostró ser multirresistente, únicamente $22.6 \%$ de los aislamientos lo fue a un grupo de antibióticos. La multirresistencia más co- mún en este grupo de hospitales se presentó contra tres grupos de antibióticos (cuadro V).

Vigilancia de la resistencia bacteriana a los antibióticos y del uso de estos fármacos

Los seis HINSCM estudiados poseían una infraestructura básica similar en lo referente al comité de infecciones, al laboratorio de diagnóstico microbiológico y a la farmacia. Cuatro instituciones (B, C, D y F) contaban con un comité de uso de antibióticos que atendía el seguimiento de la resistencia bacteriana y participaba en la selección de antibióticos para la farmacia del hospital; emitía lineamientos para su uso adecuado, autorizaba la dispensación de antibióticos de uso restringido y mantenía actualizado al cuerpo médico sobre el perfil de resistencia de los gérmenes nosocomiales. En los otros dos hospitales existía al menos un infectólogo responsable de estas funciones, pero no de la selección de antibióticos por el hospital.

En los seis hospitales existía un comité de infecciones que emprendía la detección de la infección con mayor o menor precisión, dependiendo de la capacitación del personal de enfermería y de la disponibili- 
Cuadro IV

\section{Resistencia de los géneros bacterianos por antibiótico, por Gram positivas y negativas, en Seis hospitales. Ciudad de México, DF, 1995}

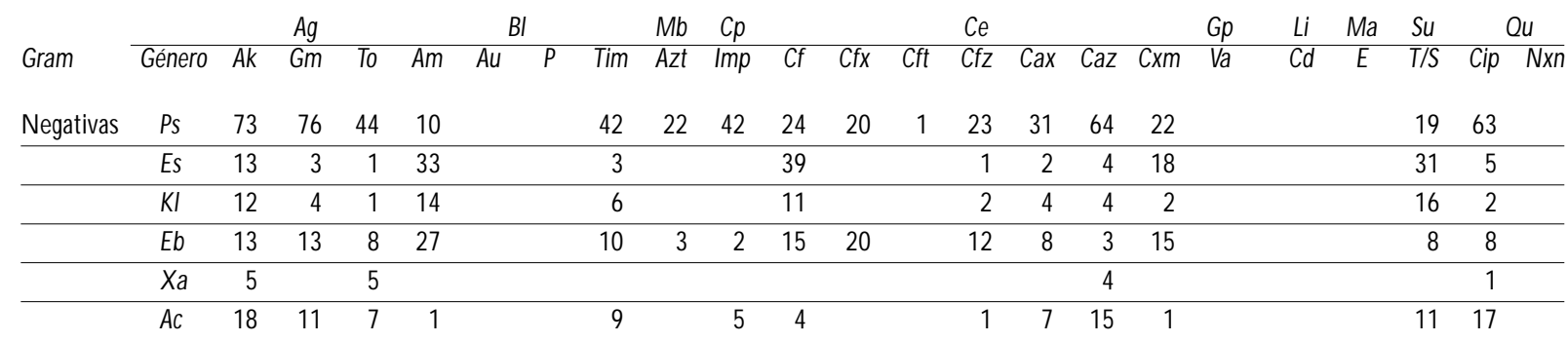

\begin{tabular}{rrrrrrrrrrrrrrrr} 
Positivas & Sta & 49 & 45 & 25 & 55 & 29 & 29 & 21 & 5 & 35 & 43 & 34 & 26 & 5 \\
\hline & EC & 2 & 10 & 3 & 7 & 4 & 8 & 1 & & 6 & 14 & 5 & 14 & 3 \\
\hline Str & & 15 & 1 & 4 & 1 & 3 & 2 & 2 & 1 & & 1
\end{tabular}

Grupos de antibióticos:Ag, amino glucósidos; Bl, betalactámicos; $\mathrm{Mb}$, mono bactámicos; $\mathrm{Cp}$, carbapenems; $\mathrm{Ce}$, cefalosporinas; $\mathrm{G} p$, glucopéptido; Li, lincosaminas; Ma, macrólidos; Su, sulfonamidas; Q u, quinolonas

Antibióticos:Ak, amikacina, G m, gentamicina;To, tobramicina;Am, ampicilina;Au,A moxicilina/ac.clavulánico; P, penicilina;T im, ticarcilina-Ac, clavulánico;Azt,

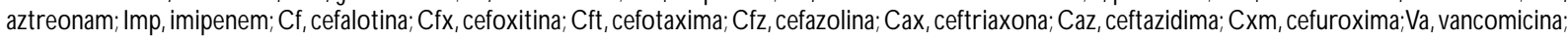
$\mathrm{Cd}$, clindamicina; E, eritromicina; $\mathrm{T} / \mathrm{S}$, trimetorpim-sulfametoxazol; Cip, ciprofloxacina; $\mathrm{N}$ xn, norfloxacina

G éneros: Ps, Pseudomonas; Es, Escherichia; KI, Klebsiella; Eb, Enterobacter; Xa,Xanthomonas;Ac, Acinetobacter; Sta, Staphylococcus; Ec, Enterococcus; Str, Streptococcus

\section{Cuadro V

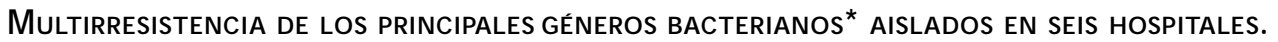 Ciudad de México, DF, 1995}

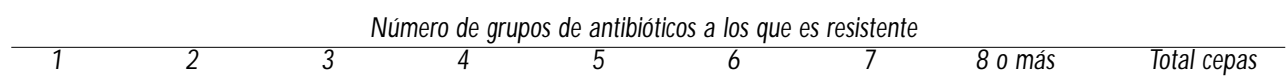

Géneros Gram negativos

\begin{tabular}{lrrrrrrrr} 
Acinetobacter & 7 & 4 & 9 & 8 & 2 & 30 & 44 \\
\hline Enterobacter & 18 & 13 & 7 & 4 & 1 & 1 & 72 \\
\hline Escherichia & 28 & 23 & 14 & 7 & & & 38 \\
\hline Klebsiella & 22 & 6 & 8 & 2 & & & 104 \\
\hline Pseudomonas & 5 & 12 & 29 & 43 & 14 & 1 & 5
\end{tabular}

Géneros Gram positivos

\begin{tabular}{lrrrrrrrrr} 
Enterococcus & 2 & 11 & 5 & 4 & 2 & 2 & 2 & 28 \\
\hline Staphylococcus & 25 & 27 & 38 & 28 & 12 & 6 & 9 & 5 \\
\hline Streptococcus & 1 & & 5 & & & & & 6 \\
\hline Total & & & & & & &
\end{tabular}

\begin{tabular}{lccccccccc} 
Total cepas R & 108 & 96 & 120 & 96 & 31 & 10 & 11 & 5 & 477 \\
\hline \% de multirresistentes & 22.6 & 20.1 & 25.2 & 20.1 & 6.49 & 2.09 & 2.3 & 1.04 & 100
\end{tabular}

* Responsables de $80 \%$ de la resistencia

N ota: Beta lactámicos y cefalosporinas se consideraron como un solo grupo en el caso de los Gram (-) 
dad de supervisión por un infectólogo. Los hospitales A, D y E no hacían muestreos representativos de las infecciones nosocomiales.

La identificación de las muestras en el laboratorio de diagnóstico microbiológico era tanto automatizada como manual. La sensibilidad a los antimicrobianos se efectuaba con equipos automatizados y con las técnicas de Kirby Bauer y concentración inhibitoria mínima en forma manual. La batería de antibióticos para valorar la sensibilidad de los aislamientos bacterianos fue distinta en cada laboratorio y dentro de un laboratorio de época en época. El control de calidad mostró ser muy variable. Dos de los seis laboratorios disponían de un programa interno de control de calidad, y de éstos, sólo uno se sometía a control de calidad externo. Ninguna institución contaba con un programa integral de vigilancia del uso de antibióticos y de la evolución de la resistencia de los gérmenes nosocomiales a estos fármacos.

\section{Discusión}

\section{Uso de antibióticos}

En dos hospitales se encontró el número más reducido de grupos de antibióticos disponibles en farmacia, pero con consumos totales distintos; en un caso se dispensaban 15 antibióticos/y se consumían 133.4 DDD/100 cd, mientras que en el otro se dispensaban 18/y se consumían 43.9 DDD/100 cd. El número de antibióticos dispensados en los otros cuatro hospitales fue alrededor de 20. En cuanto al consumo total de antibióticos, los dos hospitales con los DDD/100 cd más altos fueron B y D. La resistencia observada contra un antibiótico, no siempre fue consistente con el nivel de consumo del mismo.

Los datos sobre uso de antibióticos no representan cifras reales de consumo, ya que en todos los hospitales los únicos registros disponibles fueron las cantidades adquiridas de cada uno, en el año de 1994.

Los hospitales con el menor número de antibióticos dispensados en la farmacia fueron instituciones cuyo Comité de Antibióticos participaba en la selección y compra de estos fármacos. Los consumos más elevados de antibióticos correspondieron a los hospitales con enfermos crónicos y, por tanto, con las estancias hospitalarias más largas.

\section{Resistencia bacteriana a los antibióticos}

Los datos aquí presentados corresponden a los primo aislamientos de la cepa respectiva por paciente. En todos los laboratorios de microbiología estudiados se usaban métodos cuantitativos para valorar la sensibi- lidad a los antibióticos, sin embargo sólo un laboratorio registraba $100 \%$ de sus datos cuantitativamente (B), el resto lo hacía de forma mixta y un hospital lo hacía cualitativamente (A). Por esta razón, en este estudio se reporta la sensibilidad de las cepas como resistentes (R) o sensibles (S). Ninguno de los seis hospitales evaluaba la sensibilidad bacteriana ante la totalidad de los antibióticos que se consumían en su institución.

Fue notoria la predominancia de Gram negativos entre los géneros resistentes a los antibióticos en los hospitales estudiados, representados por: Pseudomonas, Enterobacter, Escherichia, Acinetobacter, Klebsiella y Xanthomonas, con resistencia mayor contra: Ak, Gm, Cip, ceftazidima (Caz), Cf, Am y T/S, principalmente. El género Gram negativo resistente, con más amplia distribución, fue Pseudomonas, cuya resistencia era mayor a Ak, Gm y Cip, y menor contra cefazolina (Cfz) y cefoxitina (Fox).

Los géneros Gram positivos más resistentes en este grupo de hospitales fueron: Staphylococcus, Enterococcus y Streptococcus, que presentaron resistencia fundamentalmente contra penicilina $(\mathrm{P}), \mathrm{Am}$, eritromicina (E), Gm, Cf y Cip. El género Gram positivo más resistente fue Staphylococcus, presente en seis instituciones; con mayor resistencia contra P, Am y E; y menor a vancomicina $\mathrm{V}$ y norfloxacina $(\mathrm{Nxn})$.

Respecto a la resistencia por grupo de antibióticos, los beta lactámicos y las cefalosporinas tuvieron el mayor número de cepas resistentes, seguidos de los aminoglucósidos y las quinolonas; entre los que se detectó menor resistencia están los glucopéptidos, los nitrofuranos y los anfenicoles.

El consumo total de antibióticos en este grupo de hospitales, expresado en DDD/100 cd, varió desde 43.9 hasta 194.8; en cambio la resistencia se mantuvo alrededor de $80 \%$ en cinco de las instituciones, excepto en la F, que mostró $100 \%$ de resistencia en las cepas nosocomiales valoradas.

Es difícil estudiar la relación entre el uso de antibióticos en una institución y los niveles de resistencia observados en la misma, debido a la gran variedad de factores que la afectan, sin embargo es importante contar con datos confiables sobre estos dos parámetros para coadyuvar a la toma de decisiones tanto local como nacionalmente.

\section{Vigilancia hospitalaria}

A lo largo del estudio, pudimos identificar algunos aspectos que pueden mejorar la vigilancia en cada institución. Entre los más importantes pueden mencionarse: a) el entrenamiento de médicos y enfermeras para mejorar la identificación, el muestreo y el registro de las infecciones nosocomiales; b) la estandarización 
y la mejora continua de la calidad en el laboratorio de microbiología diagnóstica para normalizar los procedimientos de identificación, valoración de la sensibilidad a los antibióticos de los aislamientos bacterianos; así como el registro y la difusión de resultados; c) la sensibilización de los médicos sobre la importancia de manejar los antibióticos apropiados según las circunstancias, y en las dosis y tiempos necesarios para evitar un uso inapropiado; d) el establecimiento de una forma de registro de dispensación de antibióticos por paciente, en la farmacia del hospital o en piso, de fácil acceso para su consulta; e) la definición de mecanismos de seguimiento en el empleo de los antibióticos dispensados en farmacia, para lograr la cuantificación precisa de su consumo en forma periódica; f) la delimitación funcional de los comités de infecciones y de uso de antibióticos, y la promoción del intercambio de información entre ellos y entre instancias relacionadas, y g) la instrumentación de un programa integral de vigilancia del uso y de la resistencia a los antibióticos, que coordine todas las instancias involucradas, como son el cuerpo médico y de enfermería, el comité de infecciones, el comité de uso de antibióticos, el laboratorio de microbiología diagnóstica, la farmacia y las autoridades del hospital.

Los hospitales que lleven a cabo el seguimiento local del uso de antibióticos ${ }^{7,34,35}$ y vigilen la emergencia y el desarrollo de la resistencia a estos fármacos en combinaciones micoorganismo-antibiótico definidas,$^{34}$ dispondrán de elementos para modular la resistencia bacteriana con un uso más racional de los antibióticos. ${ }^{36-38}$

El presente estudio se desarrolló antes de que se emitieran lineamientos internacionales tendientes a mejorar la vigilancia del uso de antibióticos dentro de las instituciones hospitalarias. Los hospitales de los institutos nacionales de salud realizan labores de investigación y docencia que fortalecen las actividades de servicio que brindan a la comunidad. La hipótesis subyacente, de que el ser instituciones con investigadores facilitaba la toma de conciencia y el desarrollo de un programa integral de vigilancia del uso de antibióticos y de los niveles de resistencia a estos fármacos en bacterias nosocomiales, se constituyó en la tesis fundamental de este trabajo.

Es importante resaltar que el hecho de disponer de lineamientos internacionales para la vigilancia del uso de antibióticos y de la resistencia bacteriana no implica que las instituciones hospitalarias, en nuestros países, dispongan de recursos humanos, materiales y financieros suficientes para instrumentarlos. ${ }^{39}$

\section{Referencias}

1. Levy SB.The antibiotic paradox. 2nd. edition. Cambridge (MA): Perseus Publishing; 2002.

2. Buckwold FJ, Ronald AR.Antimicrobial misuse-effects and suggestions for control. J Antimicrob Chemother 1979;5:129-136.

3. Avom J, Solomon DH. Cultural and economical factors that (mis)shape antibiotic use:The nonpharmacologic basis of therapeutics. Ann Intern Med 2000;133:128-135.

4. Huges VM, D otta N . C onjugative plasmids in bacteria of the "preantibiotic" era. Nature 1983;302:725-726.

5. Behra-Miellet J, Calvet L, Mory F, Muller C, Chomarat M, Bézian MC et al. Antibiotic-resistance among anaerobic gram-negative bacilli: Lessons from a french multicentric survey. Anaerobe 2003;9:105.

6. Heinemann JA, Ankenbauer B,A mábile-C uevas CF. D o antibiotics maintain antibiotic resistance? D rug D iscov Today 2000;5:195-204.

7. Kunin CM. Evaluation of antibiotic usage:A comprehensive look at alternative approaches. Rev Infect Dis 1981;3:745-753.

8. Beaber JW, Hochhut B,W aldor MK. SO S response promotes horizontal dissemination of antibiotic resistance genes. $N$ ature 2004;427:72-74.

9. A mábile-C uevas CF, ed. Multiple drug resistant bacteria. Londres: Horizon Scientific Press; 2003.

10. MCG owan Jr JE. Antimicrobial resistance in hospital organisms and its relation to antibiotic use. Rev Infect D is 1983;5:1033-1048.

11. McG owan Jr JE. Is antimicrobial resistance in hospital microorganisms related to antibiotic use? Bull NY Acad Med 1987;63:253-268.

12. Cooke D, Salter AJ, Phillips I.Antimicrobial misuse, antibiotic policies and information resourses. J Antimicrob Chemother 1980;6:435-443. 13. Gerding D N , Larson TA, Hughes RA, W eiler M, Shankoltzer C, Peterson LR. Aminoglycoside resistance and aminoglycoside usage:Ten years of experience in one hospital. Antimicrob A gents $C$ hemother 1991;35:1284-1290.

14. Mutnick AH, Rhomberg PR, Sader HS, Jones RN . Antimicrobial usage and resistance trend relationships from the MYSTIC programme in N orth America. J Antimicrob Chemother 2004:53:290-296.

15. Lipsitch M, Bergstrom CT, Levin BR.The epidemiology of antibiotic resistance in hospitals: Paradoxes and prescriptions. Proc N atl Acad Sci USA 2000;97:1938-1943.

16. Lesch CA, Itokaszu GS, D anziger LH. Multi-hospital analysis of antimicrobial usage and resistance trends. Diagn Microbiol Infect Dis 2001;41:149-154.

17. Rubin MA, Samore MH.Antimicrobial use and resistance. Curr Infect Dis Rep 2002; 4:491-497.

18. Levin BR, Lipsitch M, Perrot V, Schrag S,Antia R, Simonsen L et al.The population genetics of antibiotic resistance. $C$ lin Infect $D$ is 1997;24 (Suppl 1):S9-S16.

19. Monnet DL,Archibald LK, Phillips L, Tenover FC, MCG owan JE Jr, Gaynes RP.Antimicrobial use and resistance in eight US hospitals: Complexities of analysis and modeling. Infect Control Hosp Epidemiol 1998;19:388-394.

20. Guillemot D. Antibiotic use in humans and bacterial resistance. Curr 0 pin Microbiol 1999;2:494-498.

21. López-Lozano JM, Monnet DL,Yague A, Burgos A, G onzalo N, Campillo $P$ et al. Modeling and forecasting antimicrobial resístance and its dynamic relationship to antimicrobial use:A time series analysis. Int J Antimicrob Agents 2000;14:21-31. 
22. Monnet DL, López-Lozano JM, Campillos P, Burgos A,Yague A, Gonzalo N . Making sense of antimicrobial use and resistance surveillance data:Application of ARIMA and transfer function models. Eur Soc Clin Microbiol Infect D is 2001;7(Suppl 5):S29-S36. 23. Lipsitch M, Samore MH.Antimicrobial use and antimicrobial resistance:A population perspective. Emerg Infect $D$ is 2002;8:347-354. 24. Shlaes D M, Dale N, Gerding MD, Joseph F, John Jr MD, W illiam A et al. Society for Health $C$ are Epidemiology of America and Infectious Diseases Society of America Joint C ommittee on the Prevention of Antimicrobial resistance: $G$ uidelines for the prevention of antimicrobial resistance in hospitals. Infect Control Hosp Epidemiol 1997;18: 275-291. 25. Patton KA. Role of JCAHO standards and clinical practice guidelines in promoting appropriate antimicrobial use. Am J Health Syst Pharm 2002;59 (Suppl 3):S16-S18.

26. United States $G$ eneral Accounting 0 ffice. Antimicrobial resistance. $D$ ata to assess public health threat from resistant bacteria are limited. GAO/HEHS/N SIAD/RCED-99-132. D isponible en: http://www.gao.gov/ archive/1999/hx99132.pdf.

27. D epartment of Health. The path of least resistance. Main report. Standing Medical Advisory Committee. Sub-group on antimicrobial resistance. Londres: D epartment of Health. Publication U nit PHLS. Headquarters 0 ffice; 1998. Disponible en: http://www.doh.gov.uk/ smacful.htm.

28. 0 rganización Panamericana de la Salud. Prevención y control de la resistencia a los antimicrobianos en las Américas. Plan estratégico de vigilancia de la resistencia a los antibióticos. O PS/HCP/HCT/139/99. Disponible en: http://www.paho.org/Spanish/HCP/HCT/EER/ PlanRegionalParaguay.doc.

29. W orld Health 0 rganization. Antibiotic resistance: Synthesis of recommendations by expert policy groups (1987-2000).

W HO .APUA,USAID.W orld Health 0 rganization; 2001. D isponible en: http://www.who.int/csr/resources/publication/drugresist/ antibiotics.pdf/.
30.W orld Health O rganization. W HO Global Strategy for Containment of Antimicrobial Resistance.W HO/CDS/CSR/DRS/2001/2/EN .

Disponible en: http://www.who.int/csr/resources/publication/drugresist/ W HO CDS CSR DRS 20012 EN/en/.

31. Mendenhall W, Beaver RJ, Beaver BM. Introducción a la probabilidad y estadística. México:Thomson Learning; 2000: 248.

32.ABC Calc 1.82 .European Study Group on Antibiotic Policies. ESGAP. D isponible en: www.escmid.org/sgwp.esgap2/ esgap_abc_calc_introduc.html.

33. Thomson PLM. Diccionario de especialidades farmacéuticas.Edición 48. México, DF: Ediciones PLM; 2002.

34. Gaynes R. The impact of antimicrobial use on the emergence of antimicrobial-resistant bacteria in hospitals. Infect D is C lin N orth Am 1997;11:757-765.

35. Medina-C uevas F, N avarrete- $N$ avarro S,Avila-Figueroa C , SantosPreciado JI. FARMAC programa diseñado para vigilar la prescripción de antimicrobianos en hospitales. Gac Med Mex 2000;136:107-111. 36. Calva J], N iebla-Pérez A, Rodríguez-Lemoine V, Santos Jl, A mábileCuevas CF.Antibiotic usage and antibiotic resistance in Latin A merica. En:A mábile-Cuevas CF, comp. Antibiotic resistance: From molecular basics to therapeutic options. N ueva York: Chapman \& Hall; 1996:73-97. 37. G ums JG, Yancey RW Jr, Hamilton CA, Kubilis PS.A randomized, prospective study measuring outcomes after antibiotic therapy intervention by a multidisciplinary consult team. Pharmacotherapy 1999;19:1369-1377.

38. Food and D rugs Administration. D epartment of Health and Human Services. Antimicrobial resistance as a Public Health Issue. Henney JE. Commissioner of Food and D rugs. FDA. Department of Health and Human Services; 2000. D isponible en: http:/www.hhs.gov/asl/testify/ t000920c.html.

39. Goldmann DA, Huskins W C. C ontrol of nosocomial antimicrobialresistant bacteria:A strategic priority for hospitals worldwide. Clin Infect Dis 1997;24(Suppl 1):S139-S145. 